QUARTERLY OF APPLIED MATHEMATICS

VOLUME LXVI, NUMBER 3

SEPTEMBER 2008, PAGES 595-605

S 0033-569X(08)01128-1

Article electronically published on July 2, 2008

\title{
SOME REMARKS ON THE NUMERICAL APPROXIMATION OF STOCHASTIC DIFFERENTIAL EQUATIONS
}

\author{
BY \\ BRIAN D. EWALD (Department of Mathematics, Florida State University, Room 205C, 1017 \\ Academic Way, Tallahassee, Florida 32306-4510) \\ AND \\ ROGER TÉMAM (Institute for Scientific Computing and Applied Mathematics, Indiana University, \\ Rawles Hall, Bloomington, Indiana 47405-5701)
}

\begin{abstract}
The aim of this article is to discuss the convergence of some numerical stochastic schemes in geophysical fluid dynamics (GFD) and to make some remarks on the numerical analysis of stochastic differential equations (SDEs).
\end{abstract}

1. Introduction. Important work has been done in recent years on the numerical analysis of stochastic differential equations by G. N. Milstein, P. Kloeden and E. Platen, W. Rümelin, D. Talay and others; see, e.g., Mil, [KP, Rum, Tal1, Tal2, and the references therein. Nevertheless many problems remain open for the numerical analysis of stochastic differential equations (SDEs) and stochastic partial differential equations (SPDEs). Issues which are well understood and even classical in the deterministic context, such as consistency of numerical schemes, stability, convergence or order of approximations, are sometimes at the frontier of current knowledge in the stochastic context; perhaps as we will see in this article it may even be too early to raise such questions, but such questions will have to be addressed at some point in the future.

This presentation is based on the experience of the authors in their efforts to understand some numerical schemes used in geophysical fluid dynamics and to develop some higher-order schemes (schemes of order $>1$ in time).

After this brief introduction we will present some of the issues and difficulties related to the approximation of stochastic differential equations. Further comments will be made in the conclusion.

Some difficulties with the numerical analysis of SDEs/SPDEs.

We start by listing some of the difficulties encountered in the numerical analysis of SDEs and SPDEs, difficulties which will be addressed below:

Received May 15, 2007.

2000 Mathematics Subject Classification. Primary 65C20.

(C) 2008 Brown University 


\section{a) Derivation of the schemes}

Many numerical schemes used for the approximation of deterministic ordinary or partial differential equations are based on some utilization of Taylor's expansion. As we shall recall below, its stochastic analogue, the stochastic Taylor expansion, is more difficult to derive and contains many more terms, including terms which are hard to generate (to simulate).

\section{b) The consistency issue}

Checking the consistency of a scheme is checking that, if convergent, a given scheme will approximate the desired equation. Furthermore, in the stochastic case, the equation contains a stochastic integral, and, besides checking that the scheme approximates the desired equation, we need to verify whether the integral is approximated in the Itô or Stratonovich sense, or possibly some other sense.

\section{c) The issue of strong vs. weak convergence}

Two types of convergence of schemes can usually be obtained in the stochastic context. The first one, called strong convergence, corresponds to the convergence in an appropriate $L^{2}$-space. The weak convergence corresponds to the convergence of moments (integrals) of the unknown function. (This use of the term "weak", different from the more standard usage of weak to mean the convergence of distributions in probability, is perhaps unfortunate, but is standard in the numerics literature.) Strong convergence is closer from analysis and usually harder to achieve. Weak convergence is closer from the probabilistic point of view and in fact suffices in most practical applications.

\section{d) The order of convergence}

Most numerical schemes currently available are of order 1 or $1 / 2$ in time $(O(\Delta t)$ or $\left.O\left(\Delta t^{1 / 2}\right)\right)$ in the strong or weak sense; we will see that there are some fundamental obstructions to deriving higher-order schemes which would be otherwise desirable.

2. The stochastic Taylor formula. We are given a probability space $(\Omega, \mathcal{A}, P)$ with a filtration $\mathcal{F}=\left(\mathcal{F}_{t}\right)_{t \geq 0}$, and we consider a stochastic differential equation which can be written in one of the following three equivalent forms:

$$
\begin{aligned}
& d U=a(U) d t+b(U) d W \\
& \frac{d U(t, \omega)}{d t}=a(U(t, \omega))+b(U(t, \omega)) \frac{d W}{d t}(t, \omega), \omega \in \Omega, \\
& U_{t}=U_{0}+\int_{0}^{t} a\left(U_{s}\right) d s+\underline{\int_{0}^{t} b\left(U_{s}\right) d W_{s} .}
\end{aligned}
$$

Here $W$ is a Wiener process and the underlined integral is an Itô stochastic integral:

- $W$ is continuous, but not of bounded variation on any interval, and so nowhere differentiable,

- $W(t)-W(s)$ is Gaussian with mean 0 and variance $t-s$,

- $W(t)-W(s)$ is independent of the values of $W\left(s_{*}\right)$ for all 
$s_{*} \leq s$, i.e., independent of the $\sigma$-algebra $\mathcal{F}_{s}$.

Note that we will use the notation $W(s)$ and $W_{s}$ synonymously, depending on which we feel is more convenient.

Reminder: Itô vs. Stratonovich calculus. Let us recall that the Itô integral $\int_{0}^{t} F_{s} d W_{s}$ is defined as the limit in $L^{2}(\Omega, P)$ of the Riemann sums:

$$
\sum_{i}\left[(1-\lambda) F\left(t_{i-1}\right)+\lambda F\left(t_{i}\right)\right] \cdot\left[W\left(t_{i}\right)-W\left(t_{i-1}\right)\right],
$$

with $\lambda=0$, whereas the Stratonovich integral, sometimes written $\int_{0}^{t} F_{s} \bullet d W_{s}$, is obtained as the limit of the same sums (2) with $d=\frac{1}{2}$. For other values of $\lambda, 0 \leq \lambda \leq 1$, other (unusual) stochastic integrals can be obtained.

In fact in (1), $U$ can be a scalar or a vector valued function, $U(t, \omega) \in \mathbb{R}^{d}$. For $d>1$, (11) is then a system of SDEs, and such systems of SDEs appear e.g. when discretizing in space an SPDE: we can then rewrite (1) componentwise as

$$
d U^{i}=a^{i}(U) d t+\sum_{k=1}^{r} b^{i k}(U) d W^{k}, \quad 1 \leq i \leq d,
$$

where $U=\left(U^{1}, \ldots, U^{d}\right), a=\left(a^{1}, \ldots, a^{d}\right), b=\left(b^{i k}\right)_{\frac{1 \leq i \leq d}{1 \leq k \leq r}}$, each $W^{k}$ being a scalar Wiener process.

Taylor formula. We now recall the usual (deterministic) Taylor expansion and write it in a form suitable for stochastic generalization. We start with functions $U=U(t)$ and $f \circ U=f(U(t))$ and write:

$$
\begin{aligned}
& d U=a(U) d t, \\
& d f(U)=L f(U) d t, \\
& L=a \frac{d}{d U} \quad \text { if } d=1, \text { or } \quad L=a^{i} \frac{\partial}{\partial U^{i}} \quad \text { if } d>1,
\end{aligned}
$$

where the Einstein summation convention is understood (when $d>1$ ). Alternatively in integral form:

$$
f(U(T))=f(U(0))+\int_{0}^{T} L f(U(t)) d t .
$$

Then, applying this formula to $L f, L^{2} f$, etc., we obtain

$$
\begin{aligned}
& f(U(T))=f(U(0))+T L f(U(0))+\frac{T^{2}}{2} L^{2} F(U(0))+R, \\
& R=\int_{0}^{T} \int_{0}^{t} \int_{0}^{s} L^{3} f(U(r)) d r d s d t,
\end{aligned}
$$

or, with the compact notation to be used in the stochastic case:

$$
f_{T}=f_{0}+T L f_{0}+\frac{T^{2}}{2} L^{2} f_{0}+R .
$$


Stochastic Taylor formula. We now turn to the stochastic Taylor Formula. The formula which is used is based on the Itô calculus. It is more involved and it contains many more terms. We arrive at (see $\underline{\mathrm{KP}}$ ):

$$
f_{T}=\sum_{\alpha} L^{\alpha} f_{0} \cdot I_{\alpha}+\sum_{\beta} I_{\beta}\left(L^{\beta} f_{T}\right),
$$

where

$$
\begin{aligned}
& L^{0}=\frac{\partial}{\partial t}+a^{k} \frac{\partial}{\partial U^{k}}+\frac{1}{2} b^{k j} b^{l j} \frac{\partial^{2}}{\partial U_{k} \partial U_{\ell}}, \\
& L^{j}=b^{k j} \frac{\partial}{\partial U^{k}}, \quad 1 \leq j \leq d,
\end{aligned}
$$

and, for multi-indices $\alpha=\left(\alpha_{1}, \ldots, \alpha_{\ell}\right), \beta=\left(\beta_{1}, \ldots, \beta_{\ell}\right)$,

$$
L^{\alpha}=L^{\alpha_{1}} \ldots L^{\alpha_{\ell}}, I_{\alpha}=\int_{0}^{T} \ldots \int_{0}^{t_{2}} d W_{t_{1}}^{\alpha_{1}} \ldots d W_{t_{\ell}}^{\alpha_{\ell}},
$$

and

$$
I_{\beta}(g)=\int_{0}^{T} \ldots \int_{0}^{t_{2}} g\left(t_{1}\right) d W_{t_{1}}^{\beta_{1}} \ldots d W_{t_{\ell}}^{\beta_{\ell}},
$$

where $d W^{0}=d t$ and $d W^{k}$ is the $k^{\text {th }}$ component of $d W$. The Einstein summation convention is again understood in (5) for the Latin indices.

REMARK 1 . The integrals $I_{\alpha}, I_{\beta}$ are difficult to simulate, and this will be an impediment to high-order schemes. However, as we will see, a number of these integrals can be omitted if we are interested in weak convergence.

3. Strong convergence. We consider a (vector-valued) stochastic process $U$ that is a solution of a stochastic differential equation such as (11) and we consider a numerical scheme producing approximants $Y^{n}, 0 \leq n \leq N$, where $T=N \Delta t, \Delta t$ being the time mesh and $Y^{n}$ meant as an approximation of $U(n \Delta t)$, also written as $U_{n \Delta t}$.

The strong convergence of the scheme at order $\theta$ is the following $L^{2}$-convergence:

$$
E\left[\sup _{0 \leq n \leq N}\left|U_{n \Delta t}-Y^{n}\right|^{2} \mid \mathcal{F}_{0}\right]^{1 / 2}=O\left(\Delta t^{\theta}\right) .
$$

We now recall the well-known strong convergence results for the explicit and implicit Euler schemes and the so-called Heun scheme; we then present the convergence results for the stochastic Adams-Bashforth scheme following ET2.

Explicit Euler scheme. The scheme read: 1

$$
Y^{n+1}=Y^{n}+a\left(Y^{n}\right) \Delta t+b\left(Y^{n}\right) \Delta W^{n},
$$

with

$$
\Delta W^{n}=W_{t_{n+1}}-W_{t_{n}} \quad t_{n}=n \Delta t
$$

and $Y_{0}=U_{0}$.

This scheme converges strongly to the Itô solution of (11) at order $\frac{1}{2}$.

${ }^{1}$ Note that we previously used the superscript for $W$ to denote the components as e.g. in (3). Here it refers to time. This should be clear in the context, and we prefer to avoid making the notation more complicated. 
Implicit Euler scheme. The scheme reads

$$
Y^{n+1}=Y^{n}+a\left(Y^{n+1}\right) \Delta t+b\left(Y^{n}\right) \Delta W^{n},
$$

$\Delta W^{n}$ as in (77), and with again $Y^{0}=U_{0}$. This scheme also strongly converges at order $\frac{1}{2}$ to the Itô solution of (11).

Heun scheme. The Heun scheme is a predictor-corrector scheme, with predictor $\widetilde{Y}^{n+1}$ and corrector $Y^{n+1}$ defined by

$$
\begin{aligned}
\tilde{Y}^{n+1} & =Y^{n}+a\left(Y^{n}\right) \Delta t+b\left(Y^{n}\right) \Delta W^{n}, \\
Y^{n+1} & =\tilde{Y}^{n+1}+\bar{a}_{n} \Delta t+\bar{b}_{n} \Delta W^{n}, \\
\bar{a}_{n} & =\frac{1}{2}\left[a\left(Y^{n}\right)+a\left(\tilde{Y}^{n+1}\right)\right], \\
\bar{b}_{n} & =\frac{1}{2}\left[b\left(Y^{n}\right)+b\left(\tilde{Y}^{n+1}\right)\right] .
\end{aligned}
$$

Here $\Delta W^{n}$ is defined as before and again $Y^{0}=U_{0}$. This scheme strongly converges at order $\frac{1}{2}$ to the Stratonovich solution of (1) when $\Delta t \rightarrow 0$.

REMARK 2. In relation with the consistency issue, it is remarkable that there are no simple "warnings" indicating that the solutions of (6) or (8) will converge to the Itô solution of (11) whereas the solution of (9) converges to the Stratonovich solution of (1). This conclusion can only be reached by a nonstraightforward analysis or a deep intuitive insight.

A stochastic Adams-Bashforth scheme. We now turn to the properties of the stochastic Adams-Bashforth scheme. In the deterministic case, this scheme, often used in computational fluid mechanics, is as follows. For

$$
\frac{d U}{d t}=a(U)
$$

the scheme reads $\left(Y^{n} \sim U(n \Delta t)\right)$ :

$$
Y^{n+2}=Y^{n+1}+\frac{\Delta t}{2}\left[3 a\left(Y^{n+1}\right)-a\left(Y^{n}\right)\right] .
$$

With suitable definitions of $Y^{0}$ and $Y^{1}$, this explicit scheme is of second order in time, $O\left(\Delta t^{2}\right)$. By inspection of the stochastic Taylor formula at order 2, a stochastic version of this scheme has been proposed for equation (1) in [ET2]. Namely, for the equation

$$
d U=a(U) d t+b(U) d W
$$

we write

$$
\begin{gathered}
Y^{n+2}=Y^{n+1}+\left[\frac{3}{2} a\left(Y^{n+1}\right)-\frac{1}{2} a\left(Y^{n}\right)\right] \Delta t \\
-\frac{3}{2} \Delta t A_{n}\left(Y^{n}\right)+B_{n}\left(Y^{n}\right),
\end{gathered}
$$

in which

$$
A_{n}(U)=L^{j} a(U) \Delta W^{j}+\underline{L^{j_{1}} L^{j_{2}} a(U) I_{\left(j_{1}, j_{2}\right)}},
$$


where the random increments (e.g., $\left.I_{\left(j_{1}, j_{2}\right)}\right)$ are from time $t_{n}=n \Delta t$ to $t_{n+1}=(n+1) \Delta t$, and

$$
\begin{aligned}
B_{n}(U)= & b^{j}(U) \Delta W^{j}+L^{0} b^{j}(U) I_{(0, j)}+L^{j} a(U) I_{(j, 0)} \\
& +L^{j_{1}} b^{j_{2}}(U) I_{\left(j_{1}, j_{2}\right)}+\underline{L^{0} L^{j_{1}} b^{j_{2}}(U) I_{\left(0, j_{1}, j_{2}\right)}} \\
& +\underline{L^{j_{1}} L^{0} b^{j_{2}}(U) I_{\left(j_{1}, 0, j_{2}\right)}}+\underline{L^{j_{1}} L^{j_{2}} a(U) I_{\left(j_{1}, j_{2}, 0\right)}} \\
& +\underline{L^{j_{1}} L^{j_{2}} b^{j_{3}}(U) I_{\left(j_{1}, j_{2}, j_{3}\right)}}+\underline{L^{j_{1}} L^{j_{2}} L^{j_{3}} b^{j_{4}}(U) I_{\left(j_{1}, j_{2}, j_{3}, j_{4}\right)}},
\end{aligned}
$$

where the random increments are from time $t_{n}$ to $t_{n+2}$ minus those from $t_{n+1}$ to $t_{n+2}$. The role of the underlined terms will be described subsequently.

Strong convergence of the stochastic Adams-Bashforth scheme. For the SAB scheme we prove in [ET2]:

THEOREM 1. Under suitable (mild) regularity and decay assumptions on $a$ and $b$, the SAB scheme converges strongly to $U$ at order 2 as $\Delta t \rightarrow 0$, where $U$ is the solution of the SDE (1) taken in the Itô sense.

4. Weak convergence. We now discuss weak convergence of numerical schemes. The weak convergence of the scheme at order $\theta$ is the following convergence of the moments:

For every polynomial $g$,

$$
\left|E g(U(N \Delta t))-E g\left(Y^{N}\right)\right|=O\left(\Delta t^{\theta}\right) .
$$

For example it is known that the explicit and implicit Euler schemes, (5), (8) weakly converge to the Itô solution of (1) at order 1 (instead of order $\frac{1}{2}$ for the strong convergences for the same schemes). Similarly the solution to the Heun scheme weakly converges at order 1 (instead of $\frac{1}{2}$ for strong convergence). We now turn to the weak convergence of the Adams-Bashforth scheme.

Weak convergence of the stochastic Adams-Bashforth scheme. Weak convergence at order 2 can be proved for a simplified version of the scheme (SAB) where the underlined terms have been removed. That is,

$$
\begin{aligned}
Y^{n+2}= & Y^{n+1}+\Delta t\left[\frac{3}{2} a\left(Y^{n+1}\right)-\frac{1}{2} a\left(Y^{n}\right)\right] \\
& -\frac{3}{2} \Delta t \widetilde{A}_{n}\left(Y^{n}\right)+\widetilde{B}_{n}\left(Y^{n}\right),
\end{aligned}
$$

with

$$
\begin{aligned}
\tilde{A}_{n}(Y)= & L^{j} a(Y) \Delta W^{j}, \\
\tilde{B}_{n}(Y)= & b^{j}(U) \Delta W^{j}+L^{0} b^{j}(U) I_{(0, j)} \\
& +L^{j} a(U) I_{(j, 0)}+L^{j} b^{k}(U) I_{(j, k)} .
\end{aligned}
$$


Theorem 2 (Ewa $)$. The stochastic Adams-Bashforth scheme $(\widetilde{S A B})$ is weakly convergent to order 2 .

REMARK 3. Note that for $(\widetilde{S A B})$, the only integral difficult to simulate is $I_{(j, k)}$, unlike the scheme $(\mathrm{SAB})$ for which several integrals are difficult to simulate.

5. An implicit leap-frog scheme. We now consider a barotropic vorticity model studied by Cecile Penland and Prashant Sardeshmukh, namely

$$
\frac{d \zeta}{d t}=-\nabla(v \zeta)+S-r \xi-\kappa \nabla^{4} \zeta
$$

where

$$
\begin{aligned}
& \xi=\nabla^{2} \psi \text { is the vorticity, } \\
& \zeta=\xi+f \text { is the total vorticity, } f \text { the Coriolis term, } \\
& v=\text { the velocity, and } \\
& r, \kappa>0 \text { are dissipation constants. }
\end{aligned}
$$

The numerical scheme uses spherical harmonics and, writing $F$ for $-\nabla(v \zeta)$, the equations read

$$
\frac{d}{d t} \zeta_{n}^{m}=F_{n}^{m}+S_{n}^{m}-r \xi_{n}^{m}-\kappa\left[\frac{n(n+1)}{a^{2}}\right]^{2} \zeta_{n}^{m} .
$$

We write this equation in short as

$$
d U=\left[a_{1}(t, U)+a_{2}(t, U)\right] d t,
$$

with

$$
\begin{gathered}
\left(a_{1}\right)_{m, n}=F_{n}^{m}+S_{n}^{m}, \\
\left(a_{2}\right)_{m, n}=-r \xi_{n}^{m}-\kappa\left[\frac{n(n+1)}{a^{2}}\right]^{2} \zeta_{n}^{m} .
\end{gathered}
$$

The leap-frog scheme proceeds in two steps:

$$
\left\{\begin{aligned}
\tilde{Y}(t+\Delta t) & =Y(t-\Delta t)+2 \Delta t a_{1}(t, Y(t)), \\
Y(t+\Delta t) & =\tilde{Y}(t+\Delta t)+2 \Delta t a_{2}(t+\Delta t, Y(t+\Delta t)) .
\end{aligned}\right.
$$

Note that the last step is implicit in $Y(t+\Delta t)$, but $a_{2}$ is linear (diagonal) in $U$.

We add a white noise and (14) becomes

$$
d U_{t}=\left[a_{1}\left(t, U_{t}\right)+a_{2}\left(t, U_{t}\right)\right] d t+b\left(t, U_{t}\right) d W_{t} .
$$

The stochastic version of the leap-frog scheme is as follows:

$$
\begin{aligned}
& \tilde{Y}^{n+2}=Y^{n}+2 \Delta t a_{1}\left(t_{n+1}, Y^{n+1}\right)+M_{n}\left(Y^{n}\right)+M_{n+1}\left(Y^{n+1}\right), \\
& Y^{n+2}=\tilde{Y}_{n+2}+2 \Delta t a_{2}\left(t_{n+2}, Y^{n+2}\right),
\end{aligned}
$$

with

$$
M_{n}(y)=b^{j}\left(t_{n}, y\right) \Delta W_{n}^{j}+\underline{\underline{b b^{\prime}\left(t_{n}, Y\right) I_{(1,1), n}}} .
$$


Theorem 3 (ET2]). The scheme is strongly convergent at order one. That is, if

$$
E\left[\left|U_{t_{1}}-Y_{1}\right|^{2} \mid \mathcal{F}_{0}\right]^{1 / 2} \leq C \Delta t,
$$

then

$$
E\left[\sup _{0 \leq n \leq N}\left|U_{t_{n}}-Y_{n}\right|^{2} \mid \mathcal{F}_{0}\right]^{1 / 2} \leq C \Delta t .
$$

For the weak convergence, the terms underlined twice coming from $M_{n}\left(Y^{n}\right)$ and $M_{n+1}\left(Y^{n+1}\right)$ are omitted.

We then have

Theorem 4 (Ewa $)$. If $Y_{1}$ is chosen such that

$$
\left|E g\left(U_{1}\right)-E g\left(Y^{1}\right)\right|=O(\Delta t),
$$

for each polynomial $g$, then

$$
\left|E g\left(U_{N}\right)-E g\left(Y^{N}\right)\right|=O(\Delta t) .
$$

We now conclude with a few remarks.

REMARK 4 (Proof of weak convergence). The weak convergence results are proven by comparing the actual scheme with some version of the stochastic Taylor scheme for which weak convergence is proven in the book of Kloeden and Platen $[\mathrm{KP}$. In short, writing the stochastic Taylor formula

$$
f_{T}=\sum_{\alpha} L^{\alpha} f_{0} \cdot I_{\alpha}+\sum_{\beta} I_{\beta}\left(L^{\beta} f_{T}\right),
$$

as we did before, the stochastic Taylor scheme reads

$$
Y^{n+1}=\sum_{\alpha} L^{\alpha} f_{t_{n}} \cdot I_{\alpha},\left(t_{n}, t_{n+1}\right) .
$$

Note that the proof in $[\mathrm{KP}$ relies on quite involved probabilistic arguments.

REMARK 5 (Finite Differences Issues (SPDEs)). When discretizing SPDEs in space, the coefficient of the white noise may involve a spatial derivative of the unknown function $u(t, x)=u(t, x ; \omega)$, that is, e.g., $b=b\left(t, u, \frac{\partial u}{\partial x_{1}}\right)$.

In GFD the function $b=b(u)$ may be determined experimentally, and determining the dependance of $b$ on the derivatives of $u$ may not be easy (not accurate). In certain cases, we have studied the effect of replacing the derivative(s) of $u$ by finite differences in space from the points of views of consistency and convergence (application e.g. to the Milstein scheme). The results were satisfactory and the error at the expected order. See e.g. [ET1, ET2, EPT].

REMARK 6 (Probabilistic Issues). There are probabilistic issues related to the computation of the stochastic integrals needed in some schemes, even for weak convergence.

Partial information is sufficient in some cases, and the product of Wiener processes might be replaced by simpler processes to some extent; see e.g. the book of Kloeden and Platen. 


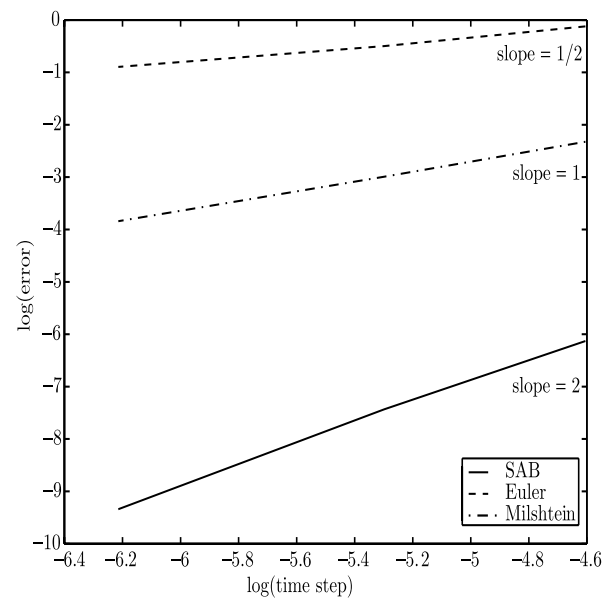

(a) Stochastic equation (17)

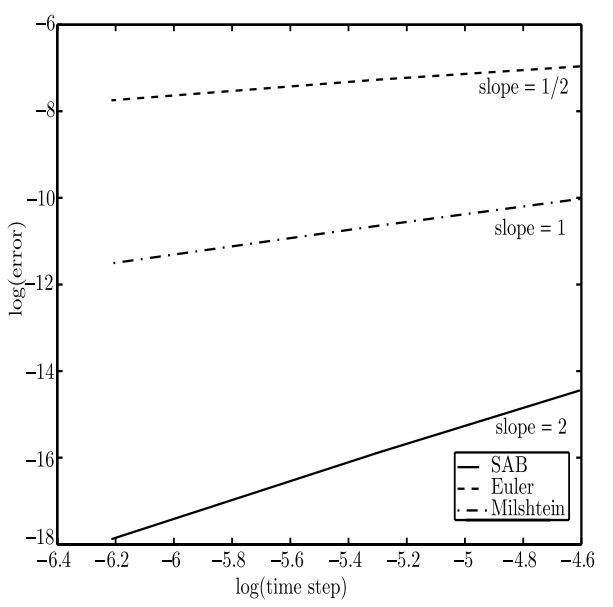

(b) Stochastic equation (18)

FIG. 1. Results obtained with the stochastic equation (17) (left) and (18) (right)

In [ET2] we address this question in a computational way: for the stochastic AdamsBashforth Scheme we approximated by a normal law the stochastic integral $I_{(0,1,1)}$ (which is difficult to generate), and this did not affect the order of the scheme for that example.

We consider the following equations:

$$
d X_{t}=\frac{1}{2} \alpha^{2} X_{t} d t+\alpha \sqrt{X_{t}^{2}-1} d W_{t},
$$

with $\alpha=1$ and $X_{0}=10$, and

$$
d X_{t}=\beta^{2} \sinh X_{t} \cosh ^{2} X_{t} d t+\beta \cosh ^{2} X_{t} d W_{t},
$$

with $\beta=1 / 10$ and $X_{0}=1 / 2$. These have the exact solutions

$$
X_{t}=\cosh \left(\alpha W_{t}+\operatorname{arccosh} X_{0}\right)
$$

and

$$
X_{t}=\operatorname{arctanh}\left(\beta W_{t}+\tanh X_{0}\right),
$$

respectively. These can be easily verified using Itô's formula and are just two of many possible examples listed in $[\mathrm{KP}$.

We computed approximate solutions $Y_{n}$ using the Euler and Milstein schemes and the $\mathrm{SAB}$ scheme from Section 3. Then we computed the following error:

$$
e=\sqrt{\mathbb{E}\left(\sup _{0 \leq n \leq N}\left|X_{n}-Y_{n}\right|^{2}\right)} .
$$

To obtain the mean value needed, we used 500 trajectories.

In the figures, the order of each scheme is given by the slope of the corresponding line. So we can see that the orders are indeed $\frac{1}{2}$ for Euler, 1 for Milstein, and 2 for the SAB of Section 3. 
Note that for the SAB scheme, the stochastic integral $I_{(0,1,1)}$ (which is difficult to generate) was approximated by a normal law. The results tend to show that this did not affect the accuracy (at least in these two cases). This point seems to raise interesting probabilistic questions.

6. Concluding remarks. (i) The proof of the results mentioned above necessitates some lengthy calculations around the stochastic Taylor formula, which need to be made "in fashion" in each case. It is clear that simplified notation and suitable "algebraic" tools should be developed to make such analysis more straightforward.

(ii) Some algorithms for computing the integrals $I_{\alpha}$ or some substitute to these integrals will be necessary.

(iii) Probabilistic tools will certainly be necessary in the numerical analysis of stochastic differential equations. Therefore, unlike deterministic numerical analysis, which starts from simple concepts such as the Taylor formula and develops into more complex analysis, the numerical analysis of the stochastic equations might proceed in the opposite way, starting from advanced concepts and ending up in practical applications.

$\begin{array}{cc}\begin{array}{c}\text { Functional Analysis (errors) } \\ \text { and more advanced concepts } \\ (\text { multilevels ... })\end{array} & \text { Advanced concep } \\ \Uparrow & \\ \text { Simple concepts: } & \\ \text { Deterministic } & \text { Applications } \\ & \text { Sumerical } \\ & \text { Analysis }\end{array}$

(iv) After the presentation of Roger Témam at the Brown Conference, Dan Crisan mentioned an alternate approach based on the so-called particle method, with work by Nicolas Victoir and collaborators (see e.g. [FV], [LV1], [LV2, etc.) which completely bypasses the problem of computing the integrals $I_{\alpha}$. Such developments are certainly very important, but, after they mature, they would imply the writing of specific totally new computing codes. This is very problematic in geophysical fluid dynamics because of the so-called legacy problem. Many expensive and sophisticated existing codes cannot be discarded, and the tendency is usually to add suitable noises to existing codes with the recognized need, advocated by e.g. Cecile Penland, to properly study the effects of these noises on the schemes, in particular to determine the type of solution (Itô or Stratonovitch integral) to which the scheme will converge.

Acknowledgments. This work was partially supported by the National Science Foundation under the grants NSF-DMS-0305110, NSF-DMS-0604235, Department of Energy under grant DOE DE-FG02-04ER63871 and by the Research Fund of Indiana University and by the Research Fund of Florida State University. 
The authors thank the referee for his/her careful reading of the manuscript.

\section{REFERENCES}

[ET2] Ewald, Brian D.; Témam, Roger, Numerical analysis of stochastic schemes in geophysics, SIAM J. Numer. Anal. 42 (2005), no. 6, 2257-2276. MR2139392 (2006b:65006)

[ET1] Ewald, B.D.; Témam, R., Analysis of stochastic numerical schemes for the evolution equations of geophysics, Appl. Math. Lett. 16 (2003), no. 8, 1223-1229. MR2015716

[EPT] Ewald, B.D.; Penland, C.; Témam, R., Accurate integration of stochastic climate models with application to El Niño, Monthly Weather Review, 132, 2004, 154-164.

[Ewa] Ewald, B.D., Error and convergence of two numerical schemes for stochastic differential equations, Numer. Methods Partial Differential Equations 22 (2006), 1247-1253. MR2248048 (2007e:65008)

[FV] Friz, Peter; Victoir, Nicolas, Approximations of the Brownian rough path with applications to stochastic analysis, Ann. Inst. H. Poincaré Probab. Statist. 41 (2005), no. 4, 703-724. MR 2144230 (2007e:60018)

[KP] Kloeden, Peter E.; Platen, Eckhard, Numerical solution of stochastic differential equations. Applications of Mathematics (New York), 23, Springer-Verlag, Berlin, 1992. MR1214374 (94b:60069)

[LV1] Lejay, Antoine; Victoir, Nicolas, On $(p, q)$-rough paths, J. Differential Equations 225 (2006), no. 1, 103-133. MR2228694 (2007i:60069)

[LV2] Lyons, Terry; Victoir, Nicolas, Cubature on Wiener space, Stochastic analysis with applications to mathematical finance. Proc. R. Soc. Lond. Ser. A Math. Phys. Eng. Sci. 460 (2004), no. 2041, 169-198. MR2052260 (2005b:35306)

[Mil] Milstein, G.N., Numerical integration of stochastic differential equations. Translated and revised from the 1988 Russian original. Mathematics and its Applications, 313, Kluwer Academic Publishers Group, Dordrecht, 1995. MR1335454 (96e:65003)

[Rum] Rümelin, W., Numerical treatment of stochastic differential equations, SIAM J. Numer. Anal. 19 (1982), no. 3, 604-613. MR656474 (83i:60075)

[Tal1] Talay, Denis, Probabilistic numerical methods for partial differential equations: Elements of analysis. Probabilistic models for nonlinear partial differential equations (Montecatini Terme 1995), 148-196, Lecture Notes in Math., 1627, Springer, Berlin, 1996. MR.1431302 (98j:60092)

[Tal2] Talay, Denis, Discretization of stochastic differential equations. Application to simulation. Stochastic numerical methods for partial differential equations. Numerical Mathematics and Advanced Applications (Jyväskä 1999), 72-86. World Sci. Publ., River Edge, NJ, 2000. MR1936171 (2003i:65010) 